\title{
DESEMPEÑO DE UN CORRELADOR HIBRIDO DE TRANSFORMACION CONJUNTA A UNA RAPIDEZ DE PROCESAMIENTO MAYOR A LA VELOCIDAD DE VIDEO,CON UN SOLO PROCESADOR DIGITAL
}

\author{
Joaquín Alfonso A. Cornejo Bautista., Yezid Torres Moreno.,María del Carmen \\ Lasprilla Alvarez
}

GOTS, Grupo de Óptica y Tratamiento de Señales, Escuela de Física, Facultad de Ciencias, Universidad Industrial de Santander,

\section{Resumen}

En este trabajo un correlador híbrido de transformación conjunta JTC de bajo costo que usa de manera complementaria las propiedades ópticas y las electrónicas, es implementado experimentalmente. Este correlador resuelve los mayores inconvenientes de un JTC óptico convencional. El JTC híbrido usa un único procesador digital de señales DSP para procesar la densidad espectral de energía conjunta JPS que es obtenida por vía óptica. La JPS de la escena y la referencia, colocadas en un modulador espacial de luz que actúa como plano de entrada, es obtenida en el plano focal imagen del procesador óptico. La adquisición digital de la JPS se hace mediante un sensor CCD que actúa como entrada al DSP. La reducción del pico central de energía de la JPS se realiza mediante un filtro óptico apodizante justo antes del sensor. Finalmente, el DSP realiza una transformación de Fourier digital de la JPS, controla todo el proceso y calcula las métricas de desempeño del correlador. Los requerimientos computacionales se reducen significativamente con la simetría hermítica de la transformada de Fourier realizada mediante el DSP para imágenes reales, esto es, que su velocidad de procesamiento lo hace hábil también para detectar objetos en escenas móviles.

Palabras clave: Correlador híbrido, Procesamiento digital de señales, Transformada de Fourier

PERFORMANCE OF A HYBRID JOINT TRANSFORM CORRELATOR WITH PROCESSING SPEED BIGGER THAN VIDEO RATE USING A SINGLE DIGITAL SIGNAL PROCESSOR

\section{Abstract}


In this paper is presented the implementation of a low cost hybrid JTC correlator that uses a combined work between their optical and electronic components. This correlator solves the major drawbacks of the conventional optical JTC. The hybrid JTC proposed here uses only one digital signal processor DSP for post-processing the joint power spectrum JPS, that is carry out in optical way. The JPS of the scene and reference, in the input plane where a spatial light modulator SLM is placed, is displayed on the focal backplane of the optical processor. The digital acquisition of the JPS is made using a CCD sensor as input to DSP. The peak energy in the center of the JPS is reduced with an apodizing filter placed just before the sensor. Finally, the DSP makes a Fourier transform of JPS, controls the complete process and finds the correlator performance. The computational requirements are notoriously reducing with the Hermitian symmetry of the Fourier transform, for real signals carried out by the DSP, that is, its processing speed does not only skillful to detect objects in still scenes.

Keywords: Hybrid Correlator, Signa digital processing, Fourier Transform.

*Para citar este artículo: Cornejo Bautista JA A., Torres Moreno Y., Lasprilla Alvarez MC.Desempeño de un correlador híbrido de transformación conjunta a una rapidez de procesamiento mayor a la velocidad de video, con un sólo procesador digital. Revista Bistua. 2017.15(1):89-101

+ Autor para el envió de correspondencia y la solicitud de las separatas: Yezid Torres Moreno Escuela de Física, Universidad Industrial de Santander.email: ytorres@uis.edu.co

Recibido: Julio 222016

Aceptado: Febrero 262017

\section{INTRODUCCIÓN}

El reconocimiento de formas por correlación juega un importante rol en aplicaciones en sistemas de vision artificial, localización de trayectorias asistida por computador, detección de cambios en el ambiente, sistemas de seguimiento, verificación de identidad, seguridad, entre otros. Esta clase de aplicaciones requieren del procesado en tiempo real rápido y la mayor precision posible. Los correladores completamente ópticos proporcionan una rápidez de respuesta suficiente, pero a menudo carecen de flexibilidad y precisión, en particular por los requerimientos en el alineamiento óptico, aspecto muy crítico por ejempo en el correlator de VanderLugt VLC (VANDERLUGT, 1964), donde se requiere de la fabricación de filtros de amplitud y fase, haciéndolo además que sea muy sensible a las vibraciones mecánicas. En el correlador puramente óptico JTC uno de los principales inconvenientes es la necesidad de utilizar un SLM de muy alta resolución para colocar la JPS de la manera más fiel posible, 
aunque al mismo tiempo es una gran ventaja porque permite realizar operaciones no lineales (JAVIDI et al. 1994). La correlación digital resuelve tales limitaciones, sin embargo, la correlación 2-dimensional es un proceso numéricamente muy grande y normalmente todas las operaciones requieren de aritmética de punto flotante que incrementa el tiempo necesario para realizarla. Los correladores digitales basados en la arquitectura de VanderLugt emplean más de un procesador digital para lograr realizar su tarea en tiempo real, por ejemplo, el sistema propuesto por Neema comprende entre 10 y 14 DSP alcanzando una rata de procesamiento de 30 frames/s para imágenes de $128 \times 128$ pixeles $^{2}$ (NEEMA et al. 2001; CAVADINI, 2001). Actualmente se debate sobre la utilidad de realizar las operaciones puramente digitales en una unidad de procesamiento gráfico GPU o bien en una unidad de procesamiento central CPU con varios núcleos de procesamiento. La experiencia reportada hasta ahora es que es posible lograr un rendimiento comparable a la de las GPU con una productividad mucho mayor en los modernos CPUs de múltiples núcleos (BORDAWEKAR et al. 2010).

(http://vsd.pennnet.com/articles/article_display.cfm?Section=ARCHI\&C=Feat\&ARTICLEID=12 4053\&KEYWORDS=DSP\%20array\&p=19, 2017) reporta un correlador híbrido que emplea ocho DSP obteniendo una velocidad de procesamiento de 25 frames/s con imágenes de entrada de $512 \times 512$ pixeles $^{2}$ con plantillas de $128 \times 128$ pixeles $^{2}$. El número elevado de procesadores digitales utilizados hace que estos dispositivos sean relativamente costosos. Un correlador VLC reportado en la literatura utiliza solamente un DSP TMS3206711 ${ }^{\mathrm{TM}}$ pero procesa aproximadamente 10 frames/s con escenas de entrada y de referencia de 256x256 pixeles $^{2}$ (GUALDRÓN et al. 2002). Todo lo anterior muestra que debe buscarse la complementación entre los procesadores ópticos y digitales puesto que combinados ofrecen la necesaria rápidez y versatilidad. En nuestro caso el JTC híbrido usa un filtrado sencillo en el espacio de Fourier a la salida del procesador óptico para controlar al origen el pico de energía y optimiza el cálculo de la transformada de Fourier para imágenes reales, a cambio de tener que usar pre-procesamiento digital para sintetizar los filtros de amplitud y fase necesarios para el VLC (WEAVER et al. 1966).

Con el fin de explicar el principio de funcionamiento en la Fig. 1(a) (WEAVER et al. 1966) se presenta el esquema del correlador puramente óptico JTC convencional; $h\left(x_{1}, y_{1}-Y / 2\right)$ y $g\left(x_{1}, y_{1}+Y / 2\right)$ en el plano de entrada, son las imágenes de referencia y la escena a correlacionar, respectivamente. La onda electromagnética de iluminación una vez colimada se propaga de izquierda a derecha. Un ejemplo del resultado de la correlación, Fig. 1b, es representado matemáticamente por:

$$
\begin{aligned}
& u_{3}\left(x_{3}, y_{3}\right)=\frac{1}{\lambda f}\left[h\left(x_{3}, y_{3}\right) * h^{*}\left(-x_{3},-y_{3}\right)+g\left(x_{3}, y_{3}\right) * g^{*}\left(-x_{3},-y_{3}\right)+h\left(x_{3}, y_{3}\right) * g^{*}\left(-x_{3},-y_{3}\right) *\right. \\
& \left.\delta\left(x_{3}, y_{3}-Y\right)+h^{*}\left(-x_{3},-y_{3}\right) * g\left(x_{3}, y_{3}\right) * \delta\left(x_{3}, y_{3}+Y\right)\right]
\end{aligned}
$$




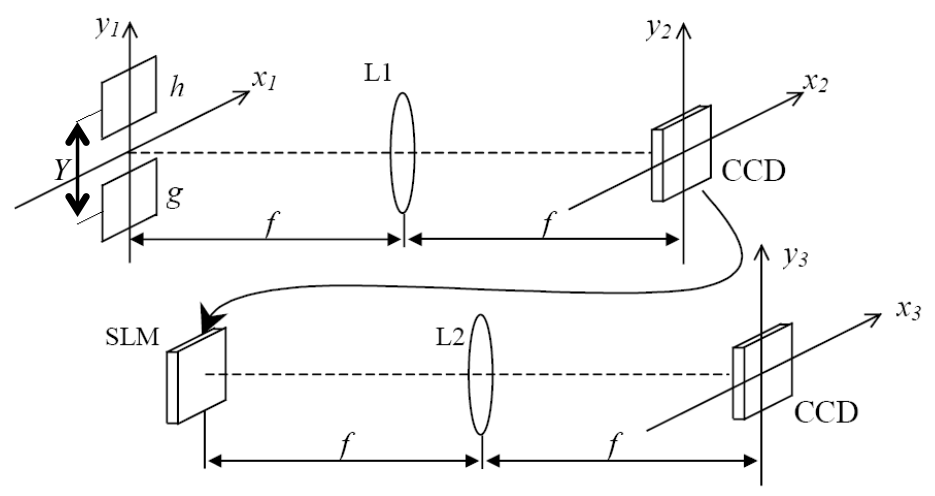

(a)

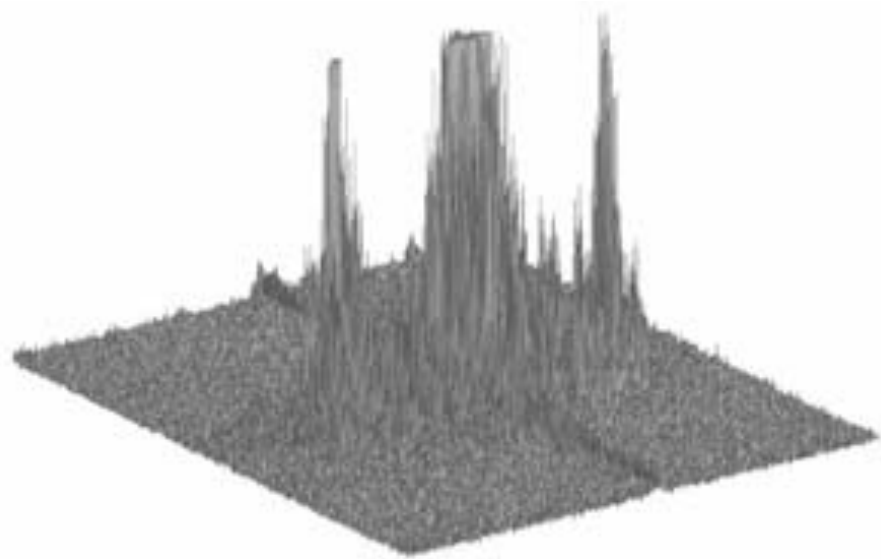

(b)

Fig. 1. (a) Dispositivo JTC óptico, la onda electromagnética de iluminación luego de colimada se propaga de izquierda a derecha. (b) Ejemplo de salida del correlador, el pico central de las autocorrelaciones ha sido truncado numéricamente.

El símbolo * representa la operación de convolución estándar y ${ }^{*}$ denota el complejo conjugado. El primer y segundo términos en la ecuación (1) corresponden a la autocorrelación tanto de la referencia como de la escena (presentes al centro del plano de salida). Los dos últimos términos corresponden a las correlaciones cruzadas posicionadas en el plano de correlación de manera simétrica respecto de su centro. $Y$, es la distancia de separación entre la referencia $h\left(x_{1}, y_{1}\right)$ y la escena $g\left(x_{1}, y_{1}\right)$ a lo largo de la dirección del eje $y_{1}$ en el plano de entrada, $\lambda$ es la longitud de onda de la radiación electromagnética coherente utilizada y $f$ es la longitud focal equivalente de la lente en el procesador óptico.

En la siguiente sección se expone el dispositivo JTC híbrido que puede realizar la correlación en tiempo real basado en el uso de un único DSP y otros elementos que hacen del mismo un dispositivo de bajo costo comparado con otras propuestas ya mencionadas.

\section{JTC híbrido basado en un dispositivo DSP}

La Fig. 2 muestra el correlador JTC híbrido; en el plano de entrada se usa una matriz de cristal liquid LCD marca CRL XGA4 ${ }^{\mathrm{TM}}$ con $1024 \times 768$ pixeles $^{2}$ en escala de grises que se comporta como modulador espacial de luz SLM. El brillo y el contraste modifican su transmitancia y ésta 
a su vez afecta significativamente el desempeño del correlador (CORNEJO et al. 2005; CORNEJO et al. 2005). Sobre la LCD se colocan la imagen de la escena a correlacionar y la imagen de referencia. La escena a correlacionar puede hacerse desfilar a la rata de transferencia máxima soportada por la LCD que es de 60 frames/s. La densidad espectral de energía conjunta JPS, es entonces calculada en tiempo real para cada pareja de imágenes sobre el sensor CCD que detecta el módulo al cuadrado de la transformada de Fourier del plano de entrada. Cuando la energía sobre la CCD es muy alta, produciendo su saturación, se usa un filtro espacial apodizador para el pico central y sí es lo suficientemente baja, es posible realizar la apodización numéricamente al momento de la adquisición antes del procesado. El sensor CCD útilizado tiene $768 \times 494$ pels $^{2}$.

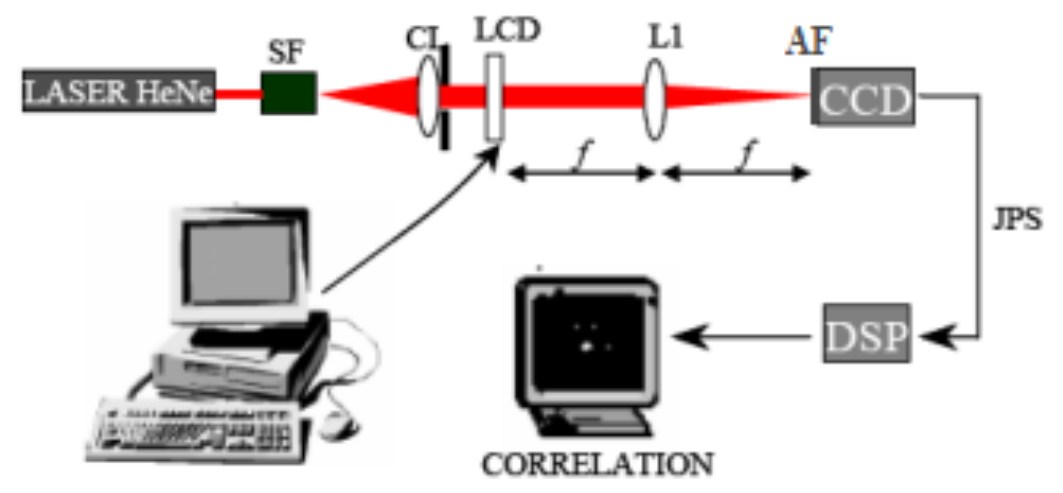

Fig. 2. Correlator JTC híbrido. SF es el filtro espacial, CL es el sistema de colimación, L1 la lente que realiza la transformación de Fourier y AF es el filtro apodizante.

La arquitectura híbrida de correlación propuesta usa un único DSP (https://training.ti.com/) para realizar la segunda etapa, en otras palabras controla la adquisición desde el sensor CCD y procesa la JPS, puede visulizar sobre un monitor el resultado de la correlación y estimar las métricas de desempeño. Una vez capturada la JSP en tiempo real en memoria externa del DSP, se realiza su transformada de Fourier obteniéndose así la salida del correlador por vía digital. La representación numérica de la correlación a la salida permite suprimir el orden central, esto es, una de las limitaciones del JTC puramente óptico puede ser superada fácilmente. La condición necesaria para evitar la superposición de los picos de correlación con el origen del plano de correlación o fondo continuo, que contiene las autocorrelaciones, es:

$$
Y>\operatorname{máx}\left\{R_{y_{1}}, E_{y_{1}}\right\}+\frac{R_{y_{1}}+E_{y_{1}}}{2} \text {, }
$$

donde $R_{y_{1}}$ y $E_{y_{1}}$ son el ancho espacial del soporte de la imagen de referencia y de la imagen de la escena, respectivamente. El resultado final, si se desea, puede ser visualizado sobre un monitor para efectos de control. La manera de calcular en el DSP es determinante de la 
velocidad de procesamiento del correlador, esto es, con el objetivo en mente de obtener 94

procesamiento en tiempo real el DSP debe ser programado para calcular eficientemente la transformada de Fourier discreta bidimensional, DFT-2D. La naturaleza real de la JPS se puede tener en cuenta y aprovechar a la hora de la programación, es decir la simetría hermitica en la ecuación (3). Dicha simetría permite calcular solamente N/2+1 transformadas de Fourier discretas unidimensionales a cambio de $\mathrm{N}$ transformaciones.

$$
F(k) \stackrel{D F T}{\leftrightarrow} f(n), \quad f(n) \in R \Rightarrow F(N-k)=F^{*}(k) .
$$

De otro lado, se programó en lenguaje assembler el cálculo de la transformada de Fourier usando el algoritmo FFT radix-2 (para señales discretas complejas) optimizado para una secuencia de 2N-puntos reales (MATUSIAK, 2017). La Fig.3 muestra un diagrama de bloques ilustrando el conjunto de procesos llevados a cabo por el DSP.

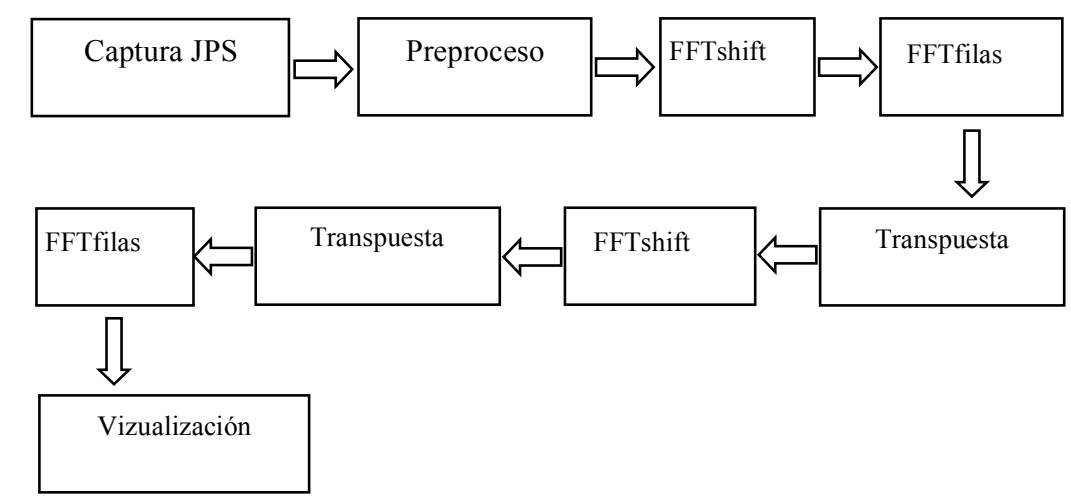

Fig. 3. Diagrama de bloques de las operaciones realizadas por el DSP.

Finalmente, el correlador híbrido realiza 31,5 correlaciones por segundo (el tiempo de ejecución contabilizado usando el reloj del DSP para la secuencia completa mostrada en la Fig. 3 es de 31,746 ms para una imagen de 256x256 pixeles ${ }^{2}$ ). Este resultado muestra que el cálculo de la DFT-2D utilizando la estratégia antes mencionada duplica la velocidad de procesamiento del correlador, en comparación con la referencia (MATUSIAK, 2017). Adicionalmente, se enfatiza en las ventajas como el tiempo de cálculo menor y la versatilidad ofrecida por la colaboración establecida, en este correlador híbrido, entre el procesador óptico y los dispositivos electrónicos. En la Fig. 4 se muestra un ejemplo de la JPS adquirida por el sensor CCD bajo el comando del DSP. La figura siguiente, Fig. 5, muestra dos ejemplos de salida del correlador. El término central dc o fondo continúo ha sido suprimido digitalmente. 


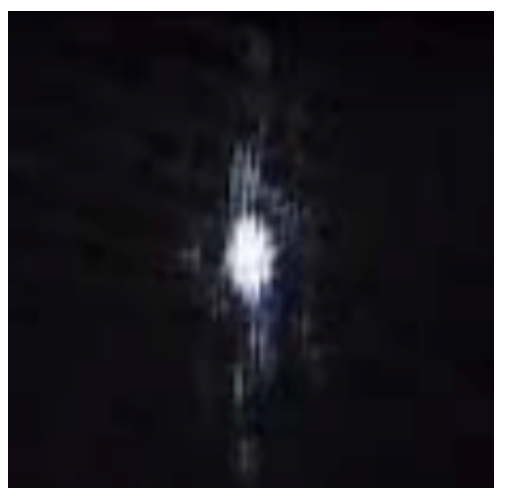

Fig. 4. JPS adquirida por el sensor CCD bajo la orden del DSP, antes de iniciar el cálculo de la DFT-2D.
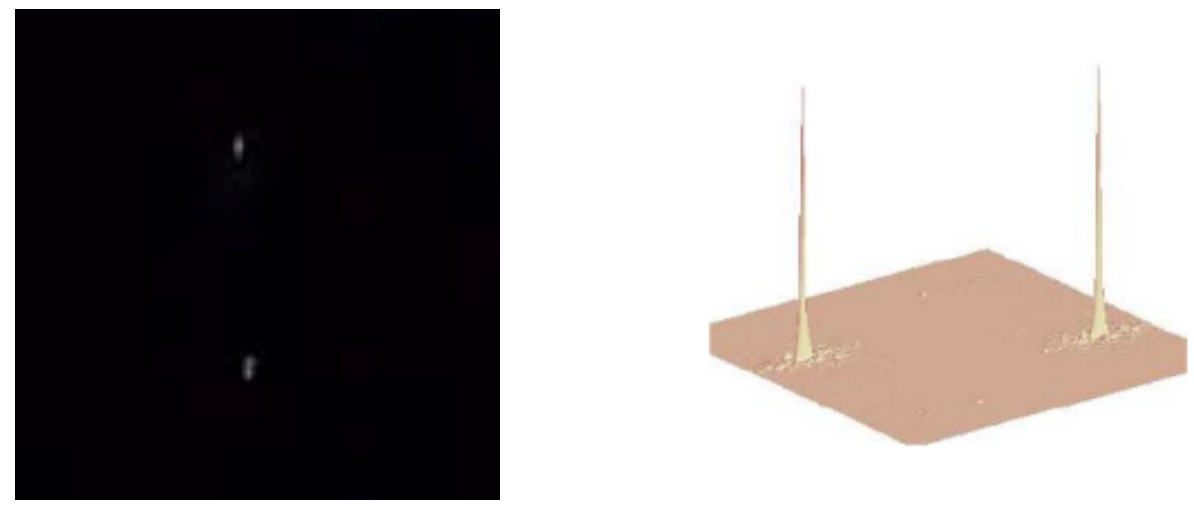

Fig. 5. Salida del correlador, a la izquierda un ejemplo de los picos de autocorrelación sobre un monitor de TV, a la derecha otro ejemplo en visualización 3D.

\section{Evaluación del desempeño del correlador}

La altura del pico, el cociente altura del pico al lóbulo lateral PSR, el cociente altura del pico a la energía de la correlación PCE, cociente señal a ruido SNR y capacidad de discriminación DC son los criterios utilizados para evaluar el desempeño del correlador (JAVIDI et al. 1994; ALAM et al. 2005). El PSR y el PCE son usados para cuantificar la estrechez del pico de correlación que indica la precisión de la localización de la escena a ser reconocida en el plano de salida (por ejemplo, cuanto mayor es el cociente PSR, más agudo es el pico y mejor es la precisión en la detección de la posición de la escena; para un PCE próximo a la unidad significa que toda la energía del plano de correlación se encuentra concentrada en el pico, que será más agudo y mejor la precisión en la posición de la escena). La Fig. 6 muestra la valoración de las métricas para cambios de escala en la escena a correlacionar. La altura del máximo en el plano de correlación cae abruptamente, cerca del $50 \%$, cuando la escala del objeto cambia hasta en $\pm 10 \%$ de la talla de la referencia (ver Fig. 6 (a)). El cociente PCE tiene un comportamiento similar a la altura del máximo del plano de correlación, ver Fig. 6 (b); es decir que cae rápidamente alrededor de un $50 \%$ para cambios en la escala de la escena de hasta $\pm 10 \%$. La variación del PCR para variaciones de hasta $\pm 10 \%$ en la escala de la escena se muestran en la Fig. 6 (c), también presenta una rápida caída del orden del $60 \%$ de su valor cuando coincide con el tamaño de la referencia. La relación señal a ruido 
SNR, que se define

96

como la relación entre la altura del pico de correlación y la desviación estándar del ruido (ruido se considera a los valores en el plano de correlación por fuera de la mitad de la altura de la anchura máxima FWHM del pico de correlación). El cociente SNR tiene el mismo significado que el usado en comunicaciones y en estimación de señales. Un valor grande de este cociente indica que la energía es muy poco dispersa fuera del pico de correlación, ruido muy bajo. En caso contrario cuando la dispersión es importante el cociente SNR toma valores pequeños; para un valor cercano a la unidad el pico es prácticamente indetectable. La Fig. 6 (d) presenta los resultados de la variación del cociente SNR, del orden del 50\%, para los mismos cambios de escala de la escena. Estos resultados indican que el correlador híbrido construido es muy sensible al cambio de escala de la escena a correlacionar, valores más allá del diez por ciento en aumento o disminución del tamaño de la escena hacen que el correlador baje abruptamente sus métricas de desempeño en más del cincuenta por ciento.

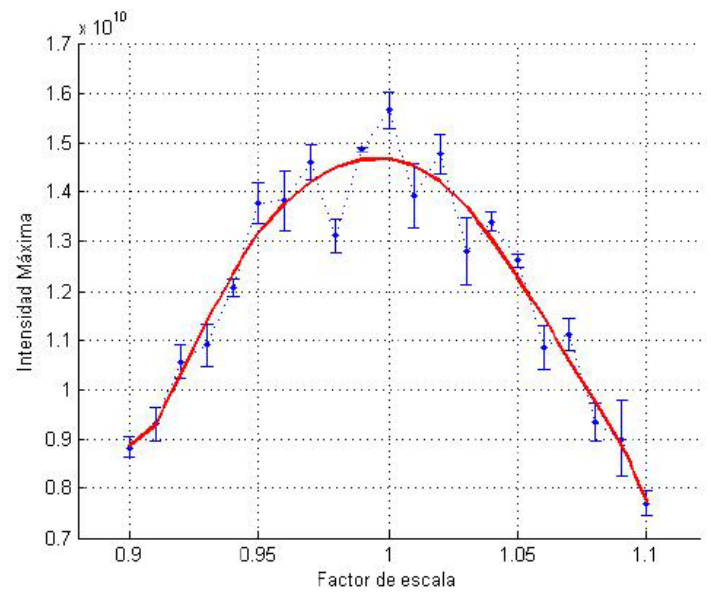

(a)

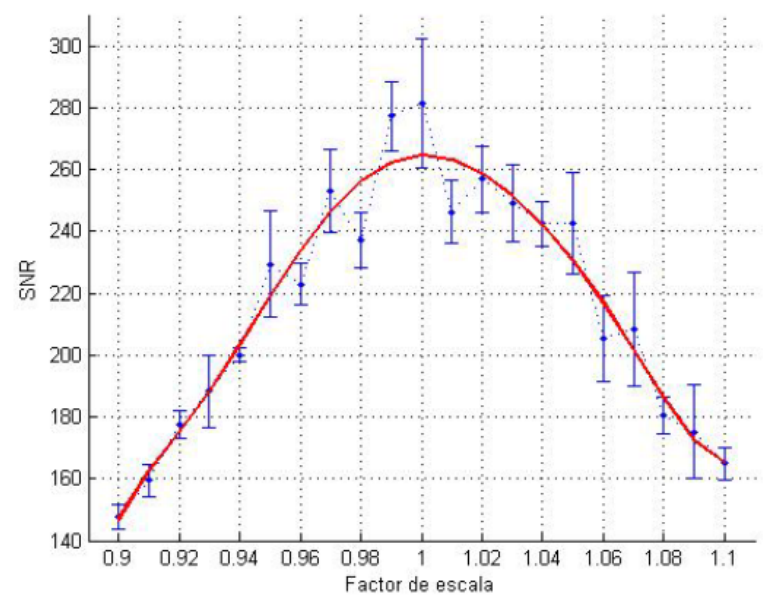

(c)

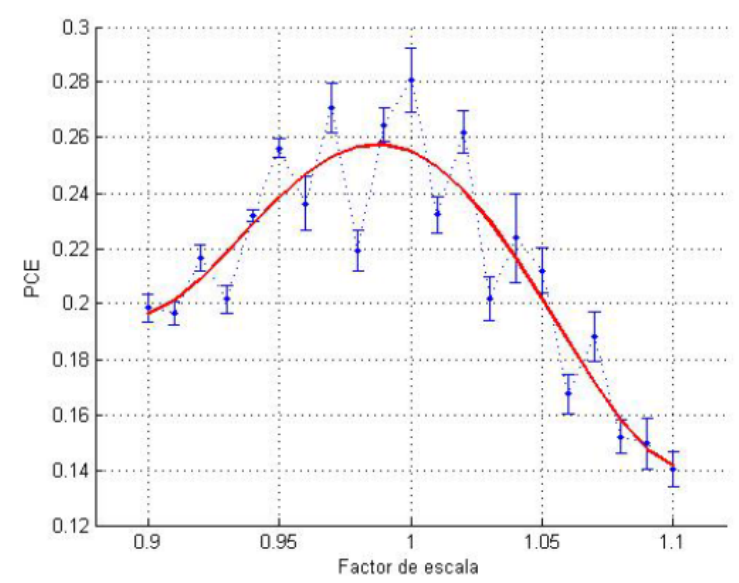

(b)

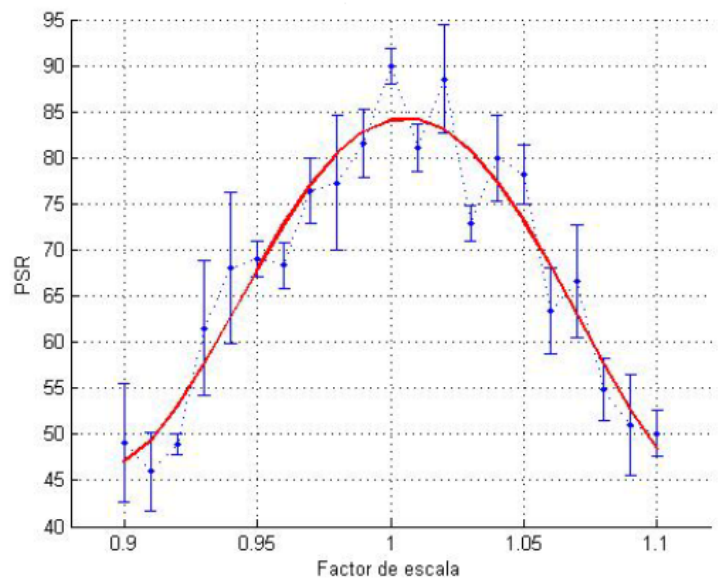

(d)

Fig. 6. (a) Máximo del pico de correlación (b) PCE (c) PSR y (d) SNR, todos ellos versus el factor de escala de la escena respecto de la referencia. 
Con el fin de evaluar el desempeño del correlador híbrido a variaciones de rotación de la escena, se calculan los valores de las diferentes métricas para rotaciones sucesivas de la escena respecto de la posición inicial de la referencia. La Fig. 7 (a) muestra como la altura del pico de correlación cae más del $15 \%$ con sólo una rotación de un grado. Al mismo tiempo, el cociente PCE cae alrededor del 12\%, ver Fig. 7 (b), para el PSR es prácticamente del mismo valor, Fig. 7 (c) y finalmente para el SNR es del orden del 7\%, ver Fig. 7 (d). Para la máxima rotación evaluada, es decir $10^{\circ}$, la pérdida abrupta de las diferentes métricas llega a ser del orden del $50 \%$ o más, excepto para el cociente SNR que llega al $20 \%$. El correlador híbrido es altamente sensible a la rotación de la escena frente a la referencia.
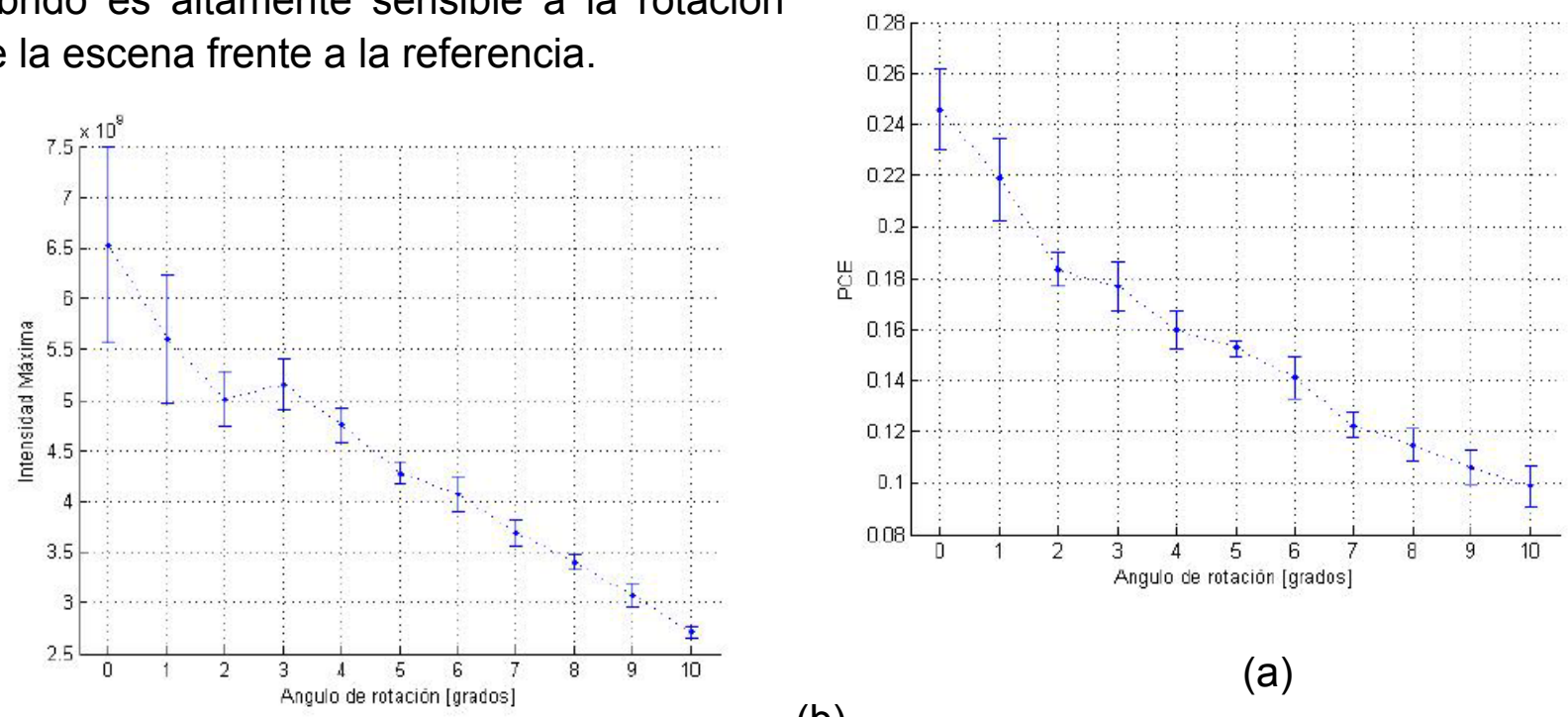

(a)

(b)
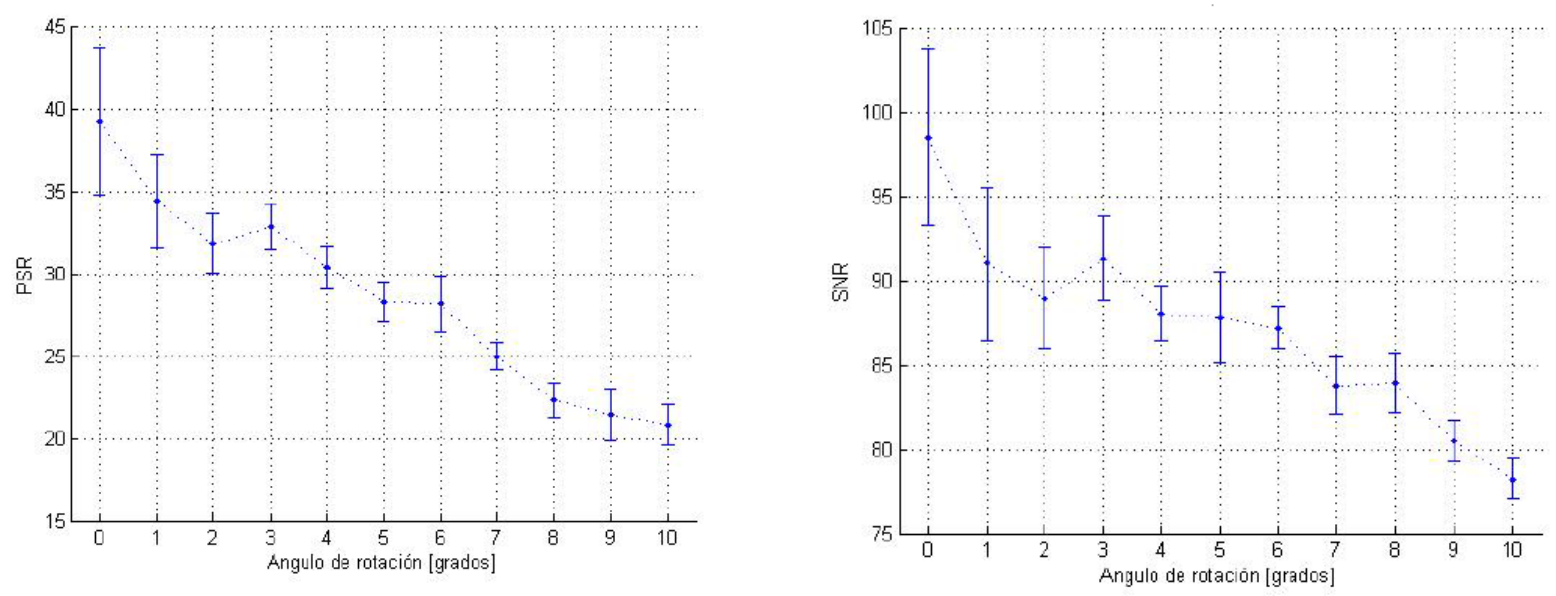

(c)

(d)

Fig. 7. (a) Máximo del pico de correlación, (b) PCE, (c) PSR y (d) SNR. Todos ellos versus el ángulo de rotación de la escena respecto de la referencia.

Para valorar la inmunidad del correlador al ruido en el plano de entrada, se agrega ruido aditivo Gaussiano blanco a la escena a correlacionar. La Fig. 8 muestra los resultados para las diferentes métricas; se puede apreciar el abrupto descenso del desempeño cuando la varianza del ruido apenas es del 10\% (para efectos de interpretación, la varianza es 
normalizada a la unidad).

98

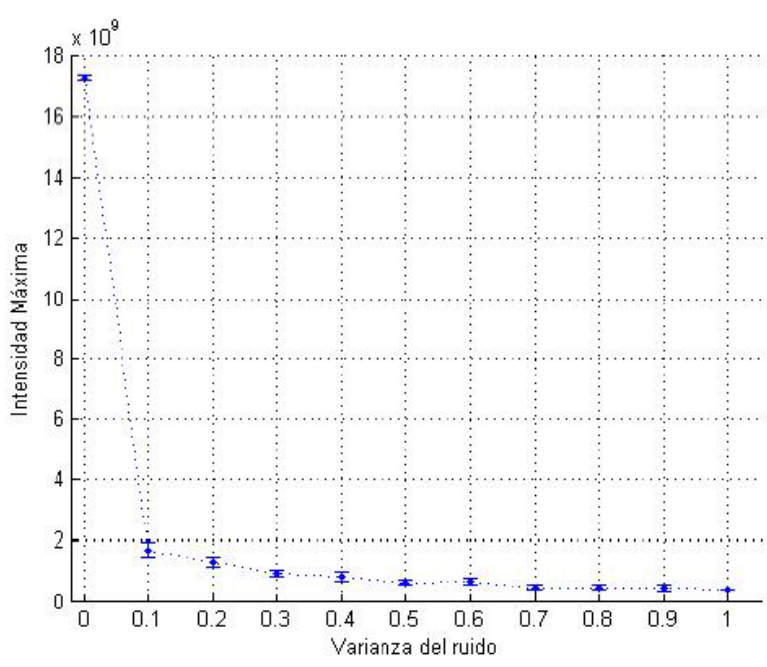

(a)

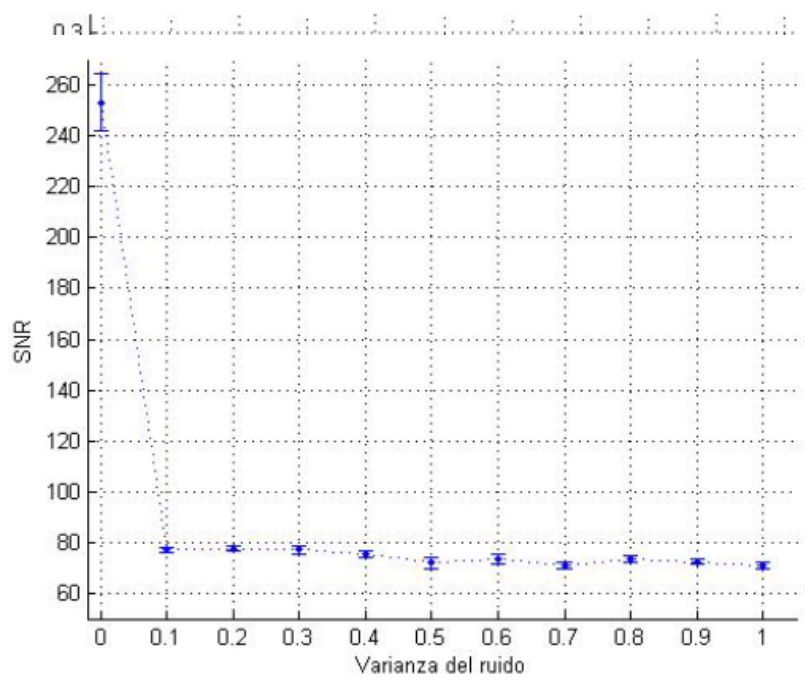

(b)

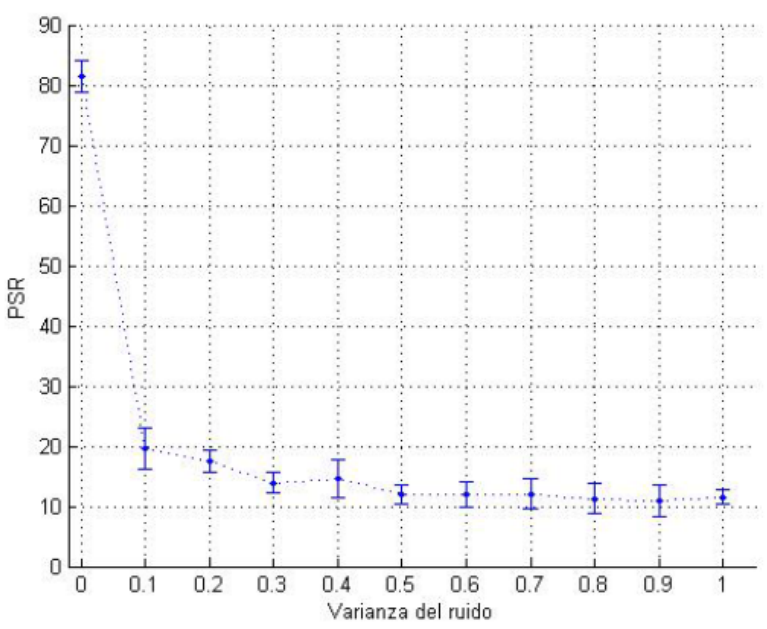

(d)

(c)

Fig. 8. (a) Máximo del pico de correlación, (b) PCE, (c) PSR y (d) SNR. Todos ellos versus la varianza normalizada del ruido aditivo blanco Gaussiano en la escena en el plano de entrada del correlador.

La Fig. 9 muestra a la derecha el plano de salida del correlador cuando la varianza normalizada del ruido aditivo Gaussiano blanco en la escena colocada en el plano de entrada alcanza el $10 \%$, a la izquierda los picos de correlación a la salida del correlador en ausencia de ruido.
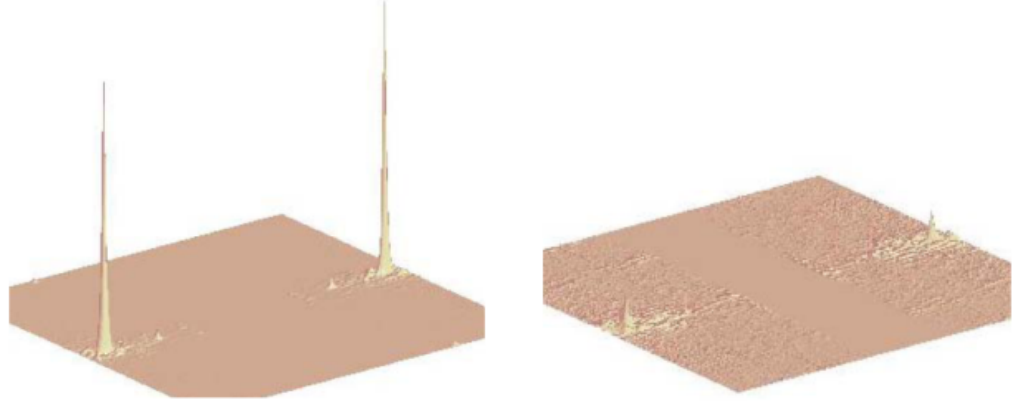

Fig. 9. Plano de salida del correlador. Izquierda cuando no hay ruido en la escena colocada en el plano de entrada. Derecha con una varianza normalizada del $10 \%$ del ruido aditivo Gaussiano blanco en la escena colocada en el plano de entrada del correlador.

El correlador híbrido tiene el desempeño típico de un correlador clásico JTC (HORNER, 
1992). La capacidad de discriminación DC, se define como (JAVIDI et al. 1994):

99

$$
D C=\left|1-\frac{C C}{A C}\right|,
$$

donde CC y AC son los máximos de la correlación cruzada y de la autocorrelación respectivamente. Cuanto más cercano a cero DC, menor es la capacidad de discriminación del correlador. En la Fig. 10 se presentan los objetos utilizados en el plano de entrada para medir DC así como la salida del correlador híbrido.
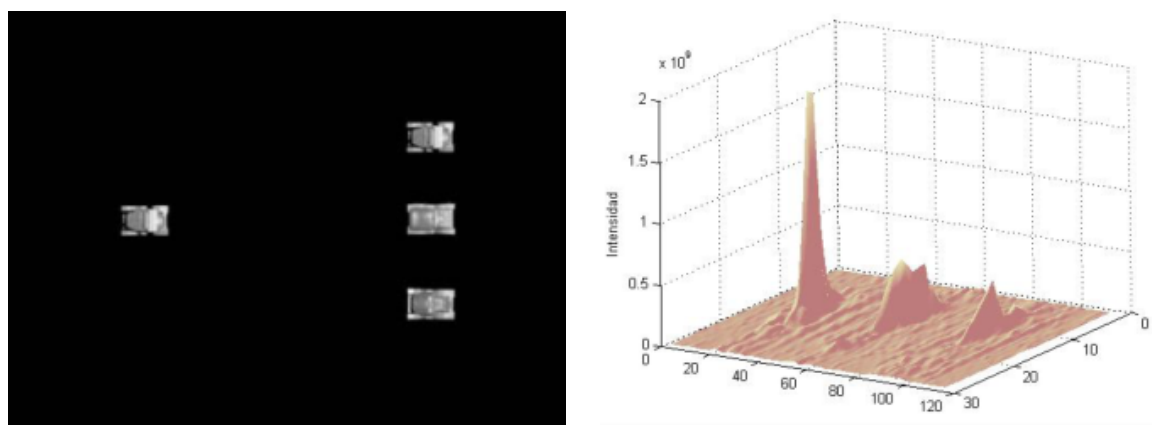

Fig. 10. Capacidad de discriminación del correlador híbrido construido. Izquierda, escena en el plano de entrada del correlador. Derecha, plano de salida del correlador con los picos de correlación cruzada (centro y derecha) y autocorrelación (izquierda).

La capacidad de discriminación del correlador híbrido se estima en $0.694 \pm 0.022$, es decir de alrededor del $69 \%$.

\section{Conclusiones}

Un correlador híbrido, óptico-digital, de transformada de Fourier conjunta JTC ha sido implementado con el uso de un único procesador DSP, a diferencia de otros que trabajan con varios procesadores alcanza un desempeño superior en velocidad de procesamiento y resolución. El JTC híbrido es capaz de realizar correlaciones a una taza de 31,5 correlaciones/s con una densidad espectral de energía JPS de 256x256 pixeles ${ }^{2}$ de resolución en el plano de entrada del DSP. El plano de entrada al correlador utiliza la máxima resolución del SLM, $1024 \times 768$ pixeles $^{2}$. En este correlador híbrido se optimiza la colaboración entre el procesador óptico y el DSP. En el DSP se programó un algoritmo muy rápido en lenguaje assembler que disminuye el tiempo de ejecución al utilizar las simetrías de las señales reales en el domino espectral. Las métricas de desempeño del correlador híbrido: altura máxima, PSR, SNR y PCE en el plano de correlación; así como su robustez al ruido en el plano de entrada son las típicas de un correlador puramente digital o puramente óptico. Su varianza a la rotación y al cambio de escala es crítica y debe tomarse muy en cuenta a la hora de las aplicaciones como por ejemplo para el reconocimiento y verificación de huellas dactilares entre otras. Aspectos asociados a la deficiencia del filtrado, como su varianza al cambio de escala, a la rotación y su baja robustez al ruido en el plano de entrada, deben ser abordados en futuros estudios, aunque ya existen propuestas de solución (JAVIDI et al. 1994; CHANG et al. 1997; Feng et al. 2002). Otro aspecto muy importante a involucrar 
en futuros estudios es la implementación de correladores híbridos usando GPU o CPU multinúcleos por su alto desempeño al momento de

100

realizar las operaciones.

\section{Agradecimientos}

Los autores expresan su agradecimiento a COLCIENCIAS (contrato 1102-05-11451 CT-189) por su apoyo financiero al igual que a la Universidad Industrial de Santander. Así como a algunos colegas anónimos por sus sugerencias al revisar el primer borrador del presente artículo.

\section{Referencias bibliogràficas}

VanderLugt A. (1964) Signal detection by complex filtering. IEEE trans. Info. Theory IT 102 : 139-145

Neema S. et al. (2001) Real Time Reconfigurable Image Recognition System. Proceedings of IEEE Conference on Instrumentation and Measurement Technology (Budapest, 2001): 350355

Cavadini M., Wosnitza M., Thaler M. Trostern G. (2001) Multiprocessor system for high resolution image correlation in real time. IEEE Transactions on Very Large Scale Integration (VLSI) Systems 9: 439-449

http://vsd.pennnet.com/articles/article display.cfm?Section=ARCHI\&C=Feat\&ARTICLEID=12 4053\&KEYWORDS=DSP\%20array\& $=19$ Vision Systems Desing Articles, DSP array upgrades optical correlation. Vision Systems Desing, April 2017

Gualdrón O. Arguello H. (2002) Correlación 2D en tiempo real- implementación mediante DSP. Proceedings of VII Simposio de Tratamiento de Señales Imágenes y Visión Artificial, (Bucaramanga, 2002): 55-60

Weaver C. Goodman J. (1966) Technique for optically convolving two functions. Appl. Opt. 5: $1248-1249$

Cornejo J. Torres Y. (2005) Hybrid joint transform correlator with DSP device - Contrast and brightness Influence. Nineth Encuentro Nacional de Óptica, Medellín, Colombia 17-21

Texas Instruments. Imaging Developer's Kit. https://training.ti.com/ May 2005

Matusiak R. (2017) Implementing fast Fourier transform algorithms of real-values sequences with the TMS320 DSP platform. Application report-Texas Instruments, August 2001 http://www.ti.com/lit/an/spra291/spra291.pdf May 2017

Cornejo J. Torres Y. (2005) Hybrid JTC using DSP technology. Eighth International 
Symposium on Laser Metrology R. Rodriguez-Vera R. Mendoza-Santoyo F. eds. Proc. SPIE 5776: $340-346$

Javidi B. Horner J. (1994) Nonlinear joint transform correlators in Real-Time Optical Information

101

Processing Academic Press, Chapter 4

Chang S. Boothroyd S. Chrostowski J. (1997) Partial rotation-invariant pattern matching and face recognition with a joint transform correlator. Appl. Opt. 36: 2380-2387

Feng L. Masahide I. Toyohiko Y. (2002) Adaptive binary joint transform correlator for image recognition. Appl. Opt. 41: 7416-7421

Alam M.S. Bal A. Horache E.-H. Goh S.-F. Loo C.H. Regula S.P. Sharma A. (2005) Metrics for evaluating the performance of joint-transform-correlation-based target recognition and tracking algorithm. Opt. Eng. 44 6: 067005 12pp

Horner J.L. (1992) Metrics for assessing pattern-recognition performance. Appl. Opt. 31 2: 165-166

Bordawekar R. Bondhugula U. Rao R. (2010) Can CPUs Match GPUs on Performance with Productivity? : Experiences with Optimizing a FLOP-intensive Application on CPUs and GPU. Technical Report IBM T. J. Watson Research Center RC $2503311 \mathrm{pp}$. 